\title{
MODELAMENTO TERMODINÂMICO DO SISTEMA NIO-CaO USANDO O MODELO QUASE-QUÍMICO DE CÉLULAS DE KAPOOR-FROHBERG-GAYE*
}

Flávio Beneduce 1

Alexandre Bellegard Farina²

\section{Resumo}

O conhecimento das características termodinâmicas do sistema $\mathrm{NiO}-\mathrm{CaO}$ é de fundamental importância no modelamento do equilíbrio metal-escória-refratário para a elaboração de ligas à base de níquel em fornos elétricos a arco assim como para o desenvolvimento de base de dados consistente para a extração de níquel de minérios lateríticos. O modelamento existente para a escória líquida, baseado no modelo quase-químico modificado por Pelton e Blander, não pode ser utilizado diretamente na base de dados SLAG usada pelo programa Thermo-Calc pois utiliza um outro modelo termodinâmico para a fase escória. $O$ presente trabalho apresenta 0 modelamento do sistema $\mathrm{NiO}-\mathrm{CaO}$ usando o modelo quase-químico de células de Kapoor-Frohberg-Gaye para a fase escória líquida. Os dados utilizados neste modelamento foram atividades das espécies químicas nas fases sólidas, composições solvus em várias temperaturas e parâmetros magnéticos da fase $\mathrm{NiO}$. A otimização foi feita com o módulo PARROT ${ }^{\circledR}$ do programa Thermo-Calc ${ }^{\circledR}$. Os resultados obtidos da otimização ajustaram-se bem aos dados experimentais apresentados na literatura. Palavras-chave: sistema $\mathrm{CaO}-\mathrm{NiO}$ system, otimização termodinâmica, KapoorFrohberg-Gaye.

\section{THERMODYNAMIC ASSESSMENT OF THE CaO-NiO SYSTEM USING THE QUASI-CHEMICAL CELL MODEL OF KAPOOR-FROHBERG-GAYE}

\section{Abstract}

The knowledge of the thermodynamic features of the $\mathrm{NiO}-\mathrm{CaO}$ system is of fundamental importance to the modelling of metal-slag-refractory equilibrium of $\mathrm{Ni}$ based alloys melting process in electric arc furnaces as well as for development a selfconsistent multi-component thermodynamic database for nickel extraction from laterite ores. The existing assessment for liquid slag, based on the quasichemical model modified by Pelton and Blander cannot be in the SLAG database used by ThermoCalc software which is based on a different thermodynamic model to slag phase. The present work presents the modelling of $\mathrm{CaO}-\mathrm{NiO}$ using the quasichemical cell model of Kapoor-Frohberg-Gaye for the liquid slag phase. The data utilized for this modelling were activities, solvus compositions as well as magnetic parameters of the phase $\mathrm{NiO}$. The optimization was performed with the PARROT ${ }^{\circledR}$ module of Thermo-Calc ${ }^{\circledR}$ software. The results obtained, the phase diagram and the activities diagrams, are in good agreement with the literature data.

Keywords: CaO-NiO system, thermodynamic assessment, Kapoor-Frohberg-Gaye.

1 Engenheiro Metalurgista, Doutor, Professor, Departamento de Engenharia Metalúrgica e de Materiais, Escola Politécnica da Universidade de São Paulo, São Paulo, São Paulo, Brasil.

2 Engenheiro Metalurgista, Doutor, Engenheiro Pesquisador Sr., Centro de Pesquisa e Desenvolvimento, Villares Metals S/A, Sumaré, São Paulo, Brasil. 


\section{INTRODUÇÃO}

Inovações e melhorias nas plantas industriais têm permitido que algumas ligas à base de níquel tradicionais (deformáveis), como por exemplo as ligas UNS N06625 (Inconel 625), UNS N07751 (Inconel X-750) e UNS N08825 (Incoloy 825), anteriormente, fundidas exclusivamente em fornos de indução à vácuo (VIM), passassem a ser fundidas em fornos elétricos à arco (EAF) ou fornos de indução ao ar. Estas inovações trouxeram grandes vantagens técnicas e econômicas à produção industrial de ligas de níquel. Entretanto, pouco é conhecido a respeito da interação metal/escória nestes sistemas já que, naturalmente, as escórias devem conter teores apreciáveis de $\mathrm{NiO}$. Consequentemente, o conhecimento das características termodinâmicas dos sistemas contendo $\mathrm{NiO}$ é de fundamental importância para o modelamento de processo envolvendo a fase escória, incluindo aí o processo de refusão por eletroescória (ESR), usual em ligas à base de Ni. Prostakova et al.[1] e Woo et al.[7] o processo pirometalúrgico de produção de $\mathrm{Ni}$ e FeNi a partir de minérios lateríticos.

Apesar de o sistema $\mathrm{NiO}-\mathrm{CaO}$ já ter sido modelado termodinamicamente por Prostakova et al.[1] utilizando, para a escória líquida, o modelo quase-químico modificado por Pelton [5-6], os dados não são compatíveis com o banco de dados SLAG, usado no Thermo-Calc ${ }^{\circledR}$ que utilizado, para a fase escória líquida, o modelo quase-químico de células de Kapoor-Frohberg-Gaye.

O objetivo do presente trabalho é realizar a otimização termodinâmica do sistema NiO$\mathrm{CaO}$ utilizando o modelo quase-químico de células de Kapoor-Frohberg-Gaye.

\section{SISTEMA NiO-CaO}

O diagrama de equilíbrio de fase do sistema pseudo-binário $\mathrm{NiO}-\mathrm{CaO}$ é um sistema eutético simples. Tikkanen (citado por Smith et al.[4]) reportou um ponto eutético na temperatura de $1720^{\circ} \mathrm{C}$ e uma fração molar de $\mathrm{CaO}$ de 0,42. Smith et al.[4] determinou o invariante na mesma concentração de $\mathrm{CaO}$ porém na temperatura de $1695^{\circ} \mathrm{C}$. Os limites de solubilidade no sólido foram determinadas por diversos pesquisadores [1,4,9] com divergências, como pode ser observado na Tabela 1.

As atividades dos componentes nas soluções sólidas foram obtidas por Raghavan [3] e Rog et al.[2]. Em ambos trabalhos é observado um forte desvio positivo da idealidade. Os dados de Rog et al. [2], como apontado por Prostakova et al. [1], sugerem limites de solubilidade no sólido muito maiores que os observados por outros autores [1,4], a ponto de não ter sido utilizado por Prostakova et al.[1] na sua otimização. A Tabela 2 apresenta as atividades utilizadas a $1313 \mathrm{~K}$ no presente trabalho incluindo os dados de Rog et al.[2]. 
Tabela 1. Limites de solubilidade no sólido do sistema NiO-CaO

\begin{tabular}{|c|c|c|c|c|c|c|}
\hline \multirow{2}{*}{$\begin{array}{c}\begin{array}{c}\mathbf{T} \\
\left({ }^{\circ} \mathbf{C}\right)\end{array} \\
1100\end{array}$} & \multicolumn{2}{|c|}{$\begin{array}{c}\text { \%NiO } \mathrm{caO} \\
\text { (mol\%) }\end{array}$} & \multirow[t]{2}{*}{$\begin{array}{c}\text { \%NiO } \\
\text { (mol\%) }\end{array}$} & \multicolumn{2}{|c|}{$\begin{array}{c}\% \mathrm{CaO}_{\mathrm{NiO}} \\
\text { (mol\%) }\end{array}$} & Ref. \\
\hline & 0,8 & & & 2,5 & & [4] \\
\hline \multirow[b]{2}{*}{1200} & 2,1 & & 96,5 & & & {$[1]$} \\
\hline & 1,2 & $2,0^{*}$ & & 3,7 & $4,0^{*}$ & \multirow[b]{2}{*}{ [4] } \\
\hline 1300 & 2,2 & $4,95^{*}$ & & 5,2 & $5,6^{*}$ & \\
\hline 1346 & 2,6 & & & 7,4 & & \multirow[b]{2}{*}{ [9] } \\
\hline 1352 & 6,6 & & & 6,7 & & \\
\hline \multirow{2}{*}{1400} & 5,5 & & 90,7 & & & [1] \\
\hline & 3,6 & $8,3^{*}$ & & 6,7 & $7,6^{*}$ & [4] \\
\hline 1410 & 7,9 & & & 8,7 & & \multirow{5}{*}{ [9] } \\
\hline 1420 & 8,2 & & & 9,0 & & \\
\hline 1423 & 8,3 & & & 9,1 & & \\
\hline \multirow{2}{*}{1481} & 5,0 & & & 12,0 & & \\
\hline & 6,6 & & & 11,0 & & \\
\hline 1485 & & $11,4^{*}$ & & & $10,2^{*}$ & \multirow[b]{2}{*}[4]{} \\
\hline 1500 & 5,8 & & & 9,3 & & \\
\hline 1524 & 6,4 & & & 14,0 & & \multirow{3}{*}{ [9] } \\
\hline \multirow{2}{*}{1527} & 6,5 & & & 16,0 & & \\
\hline & 5,4 & & & 14,0 & & \\
\hline \multirow{2}{*}{1600} & 8,4 & & & 12,6 & & {$[4]$} \\
\hline & 9,3 & & 85,2 & & & [1] \\
\hline 1695" & 11,0 & & & 17,0 & & {$[4]$} \\
\hline
\end{tabular}

Tabela 2. Atividade do $\mathrm{CaO}$ em função da fração molar de $\mathrm{CaO}$ a 1313K (ref. CaO sólido)

\begin{tabular}{ccc}
\hline $\mathbf{X}_{\mathrm{CaO}}$ & \multicolumn{2}{c}{$\mathbf{a c a O}$} \\
\cline { 2 - 3 } & Raghavan[3] & Rog et al.[2] \\
\hline 0,015 & 0,746 & 0,081 \\
\hline 0,020 & & 0,190 \\
\hline 0,050 & 0,980 & 0,310 \\
\hline 0,080 & & 0,323 \\
\hline 0,100 & 0,977 & 0,893 \\
\hline 0,150 & & 0,909 \\
\hline 0,400 & \\
\hline 0,500 & 0,979 & 0,911 \\
\hline 0,620 & & 0,909 \\
\hline 0,800 & \\
\hline 0,890 & 0,908 \\
\hline 0,900 & 0,930 \\
\hline 0,920 & 0,975 \\
\hline 0,950 & \\
\hline 0,975 & 0,954 \\
\hline 0,980 & \\
\hline
\end{tabular}

\section{MODELOS TERMODINÂMICOS UTILIZADOS}

A fase líquida (SLAG) foi modelada segundo o modelo quase-químico de células de Kapoor-Frohberg-Gaye. Este modelo considera a existência de células compostas de cátions e ânions (oxigênio) que podem ser simétricas ( $\mathrm{Ca}-\mathrm{O}-\mathrm{Ca}$ e $\mathrm{Ni}-\mathrm{O}-\mathrm{Ni}$ ) e assimétricas $(\mathrm{Ca}-\mathrm{O}-\mathrm{Ni})$. Essas células estão aleatoriamente distribuídas na solução. As energias livres de formação das células simétricas são iguais às energias livres de formação dos óxidos líquidos puros. A energia livre da célula mista (assimétrica) e a interação entre as células simétricas, que podem ser dependentes 
da composição, são parâmetros a serem otimizados. As energias associadas à formação das células simétricas são $W_{\text {NiNi }}^{0}$ e $W_{C a C a}^{0}$ e da assimétrica $W_{\text {NiCa }}^{0}$. As frações de células do sistema como $R_{N i N i}, R_{C a C a}$ e $R_{N i C a}$, onde $R_{N i N i}+R_{N i C a}+R_{C a C a}=1$. A energia livre molar da fase é avaliada pela Equação 1 [10].

$$
\begin{aligned}
&{ }^{S L A G} G=+W_{N i N i}^{O}\left(c X_{N i O}-R_{N i C a}^{O}\right)+W_{N i C a}^{o} R_{N i C a}^{O}+W_{C a C a}^{O}\left(c X_{C a O}-R_{N i C a}^{O}\right) \\
&- R T\left[\frac{a}{c}\left(c X_{N i O}+d X_{C a O}\right) \ln \left(\frac{c X_{N i O}+d X_{C a O}}{c X_{N i O}}\right)-d X_{C a O} \ln \left(\frac{d X_{C a O}}{c X_{N i O}}\right)\right] \\
&- 2 R T\left[c X_{N i O} \ln \left(\frac{c X_{N i O}}{c X_{N i O}+d X_{C a O}}\right)-d X_{C a O} \ln \left(\frac{d X_{C a O}}{c X_{N i O}+d X_{C a O}}\right)\right] \\
&+ R T\left[\left(c X_{N i O}-R_{N i C a}^{O}\right) \ln \left(\frac{c X_{N i O}-R_{N i C a}^{o}}{c X_{N i O}+d X_{C a O}}\right)+R_{N i C a}^{O} \ln \left(\frac{R_{N i C a}^{o}}{c X_{N i O}+d X_{C a O}}\right)\right] \\
&+\left(d X_{C a O}-R_{N i C a}^{O}\right) \ln \left(\frac{d X_{C a O}-R_{N i C a}^{o}}{c X_{N i O}+d X_{C a O}}\right)
\end{aligned}
$$

Os cálculos das frações de células são realizados segundo uma função de partição, a qual é dependente da energia de interação entre células $\left(E_{N i N i}^{0}, E_{N i C a}^{0}\right.$ e $\left.E_{C a C a}^{0}\right)$, são apresentados nas Equações 2 e 3.

$$
\begin{aligned}
& R_{N i C a}^{O}=\frac{\phi^{2}\left(c X_{N i O}+d X_{C a O}\right)+\sqrt{\left(\phi^{2} c X_{N i O}-\phi^{2} d X_{C a O}\right)-4 \phi^{2} c X_{N i O} d X_{C a O}}}{2\left(\phi^{2}-1\right)} \\
& R_{N i N i}^{O}=c X_{N i O}-R_{N i C a}^{O} \text { e } \quad R_{C a C a}^{O}=d X_{C a O}-R_{N i C a}^{O}
\end{aligned}
$$

Onde

$$
\phi=\left[\frac{1}{R T\left(c X_{N i O}+d X_{C a O}\right)}\right]^{2}\left(c X_{N i O} E_{N i N i}^{O}+d X_{C a O} E_{N i C a}^{O}\right)\left(c X_{N i O} E_{N i C a}^{O}+d X_{C a O} E_{C a C a}^{O}\right) \exp \left(\frac{-W_{N i C a}^{O}}{R T}\right)
$$

O modelo termodinâmico acima apresentado foi posteriormente modificado por Gaye, o qual o expandiu para avaliação de sistemas poli-aniônicos e poli-catiônicos [11]. As soluções sólidas ( $\mathrm{NiO}$ e $\mathrm{CaO}$ ) foram descritas termodinamicamente pelo modelo da solução substitucional-regular [8] descritas pelas Equações 4 a 9. Assim:

$$
\begin{aligned}
& G^{\text {fase }}={ }^{r e f} \mathrm{G}+{ }^{i d} \mathrm{G}+{ }^{e x c} \mathrm{G}+{ }^{\text {mag }} \mathrm{G} \\
& { }^{\text {ref }} \mathrm{G}^{\text {fase }}=\mathrm{x}_{\mathrm{NiO}}{ }^{\text {ref }} \mathrm{G}_{\mathrm{NiO}}+\mathrm{x}_{\mathrm{CaO}}{ }^{\text {ref }} \mathrm{G}_{\mathrm{CaO}}
\end{aligned}
$$

Equação 4

Equação 5

Onde: ${ }^{\text {ref }} \mathbf{G}_{\mathbf{i}}={ }^{\mathbf{S E R}} \mathbf{G}_{\mathbf{i}}$

$$
\begin{aligned}
& { }^{\mathrm{id}} \mathrm{G}^{\text {fase }}=\mathrm{R} \cdot \mathrm{T}\left[\mathrm{x}_{\mathrm{NiO}} \cdot \ln \mathrm{x}_{\mathrm{NiO}}+\mathrm{x}_{\mathrm{CaO}} \cdot \ln \mathrm{x}_{\mathrm{CaO}}\right] \\
& { }^{\text {exc }} \mathrm{G}^{\text {fase }}=\mathrm{x}_{\mathrm{NiO}} \cdot \mathrm{x}_{\mathrm{CaO}} \cdot L^{\text {fase }}
\end{aligned}
$$

O parâmetro de interação é descrito por um polinômio de Redlich-Kister (Equação 5). 
A Equação 10 apresenta o cálculo da contribuição do magnetismo à energia livre [8].

$$
\left.{ }^{m a g} G^{N i O}=R . T \cdot Q \tau\right) . \quad \ln \left(\beta_{0}+1\right)
$$

O parâmetro $\tau$ é o quociente entre a temperatura absoluta do sistema e a temperatura de Curie da solução $\left(\tau=T / T_{C}\right)$. A temperatura de Curie da solução $\left.\left(T_{C}\right), 0 \notin \tau\right)$ e o coeficiente $\beta_{0}$ (momento magnético médio) do $\mathrm{NiO}$ foram obtidas do trabalho de Prostakova et al.[1].

Os parâmetros termodinâmicos utilizados no presente trabalho estão relacionados na Tabela 3. Os parâmetros GS_NiO e GS_CaO (GHSER ou ${ }^{S E R} G_{i}$ ) foram obtidos dos dados do SGTE [12]. Os dados experimentais foram otimizados termodinamicamente usando a técnica do CALPHAD com a ajuda do módulo PARROT do Thermo-Calc ${ }^{\circledR}$.

\section{RESULTADOS E DISCUSSÃO}

Na Tabela 3 são apresentados os parâmetros termodinâmicos das fases SLAG, NiO e $\mathrm{CaO}$ otimizados para o sistema $\mathrm{NiO}-\mathrm{CaO}$.

Tabela 3. Parâmetros termodinâmicos utilizados no presente trabalho $(\mathrm{J} / \mathrm{mol})$.

\begin{tabular}{|c|c|c|}
\hline Fase & Parâmetro & Equação \\
\hline \multirow{5}{*}{$\begin{array}{c}\text { SLAG } \\
\text { (Líquido) }\end{array}$} & ${ }^{0} \mathrm{G}_{\mathrm{NiO}}^{\mathrm{SLAG}}$ & GS_NIO $=50662.4-22.719^{*} \mathrm{~T}-\mathrm{GI} N \mathrm{NIO}$ \\
\hline & ${ }^{0} \mathrm{G}_{\mathrm{CaO}}^{\mathrm{SLAG}}$ & GS_CAO $=80000-27.941 * T$ - GI_CAO \\
\hline & 2. ${ }^{0} \mathrm{~W}_{\mathrm{NiCa}}$ & $+\mathrm{V} 10+\mathrm{V} 11^{*} \mathrm{~T}+\mathrm{GS} \_\mathrm{NIO}+\mathrm{GS} \_\mathrm{CAO}$ \\
\hline & ${ }^{0} \mathrm{E}_{\mathrm{NiCa}}$ & V20 \\
\hline & ${ }^{1} \mathrm{E}_{\mathrm{NiCa}}$ & V21 \\
\hline \multirow{3}{*}{$\mathrm{CaO}$} & ${ }^{0} \mathrm{G}_{\mathrm{CaO}}^{\mathrm{CaO}}$ & Gl_CAO* \\
\hline & ${ }^{0} \mathrm{G}_{\mathrm{NiO}}^{\mathrm{CaO}}$ & $+\mathrm{V} 30+\mathrm{V} 31^{*} \mathrm{~T}$ \\
\hline & ${ }^{0} \mathrm{~L}_{\mathrm{CaO}, \mathrm{NiO}}^{\mathrm{CaO}}$ & V40 \\
\hline \multirow{3}{*}{$\mathrm{NiO}$} & ${ }^{0} \mathrm{G}_{\mathrm{NiO}}^{\mathrm{NiO}}$ & GS_NIO ** \\
\hline & ${ }^{0} \mathrm{G}_{\mathrm{CaO}}^{\mathrm{NiO}}$ & $+\mathrm{V} 50+\mathrm{V} 51^{\star} \mathrm{T}$ \\
\hline & ${ }^{0} \mathrm{~L}_{\mathrm{NiO}, \mathrm{CaO}}^{\mathrm{NiO}}$ & V60 \\
\hline
\end{tabular}

\footnotetext{
*298.15K $<\mathrm{T}<$ 1400K: $-648652.187+401548.94 * \mathrm{~T}^{-1}+299.652412 * \mathrm{~T}$

$-49.5234976 * T * L N(T)-.00282997392 * T^{2}+1.05894251 E-07 * T^{3}$

1400K $<\mathrm{T}<3200 \mathrm{~K}:-647266.711+295.317001 * \mathrm{~T}-49.0716256 * \mathrm{~T} * \mathrm{LN}(\mathrm{T})$

$-.00252542056 * T^{2}+2.97808752 E-08 * T^{3}$

$3200 \mathrm{~K}<\mathrm{T}<4000 \mathrm{~K}:-667160.922+404.235137 * \mathrm{~T}-62.76 * \mathrm{~T} * \mathrm{LN}(\mathrm{T})$

$* * 298.15 \mathrm{~K}<\mathrm{T}<524 \mathrm{~K}:-255476.618+297.571533 * \mathrm{~T}-.00297487 * \mathrm{~T}^{2}$

$+\mathbf{5 8 9 1 5 0} * \mathrm{~T} * *(-\mathbf{1})-\mathbf{1 4 8 0 8 3 5 0} * \mathrm{~T}^{-2}-\mathbf{4 9 . 8 8 7} * \mathrm{~T} * \mathrm{LN}(\mathrm{T})$

$524 \mathrm{~K}<\mathrm{T}<2230 \mathrm{~K}:-255476.618+297.571533 * \mathrm{~T}-.00297487 * \mathrm{~T}^{2}$

$+589150 * T^{-1}-14808350 * T^{-2}-49.887 * T * L N(T)$
}

Os resultados das variáveis otimizadas estão apresentados na Tabela 4. 
Tabela 4. Variáveis otimizadas

\begin{tabular}{|c|c|c|}
\hline Variável & $\mathbf{J} / \mathbf{m o l}$ & $\mathbf{J} /(\mathbf{K} . \mathbf{m o l})$ \\
\hline V10 & 3166923 & \\
\hline V11 & & 1531,1418 \\
\hline V20 & $-41230,22$ & \\
\hline V21 & 15646,941 & \\
\hline V30 & $-88677,570$ & $-161,13892$ \\
\hline V31 & & \\
\hline V40 & 17625,329 & $-143,10039$ \\
\hline V50 & $-513607,97$ & \\
\hline V51 & & \\
\hline V60 & 22025,283 & \\
\hline
\end{tabular}

Como pode ser observado na Figura 1, os dados experimentais de limites de solubilidade nas fases $\mathrm{NiO}$ e $\mathrm{CaO}$ de ficaram bem ajustados no diagrama de equilíbrio principalmente aos dados de Smith et al. [4]. As linhas liquidus e as solvus acima do patamar eutético puderam ser calculadas baseadas única e exclusivamente nos dados experimentais do ponto eutético, dos limites do patamar eutético e dos pontos de fusão das substâncias puras como no trabalho de Prostakova et al. [1]. De qualquer forma, uma avaliação experimental da linha liquidus é uma condição necessária para tornar a otimização mais confiável e robusta.

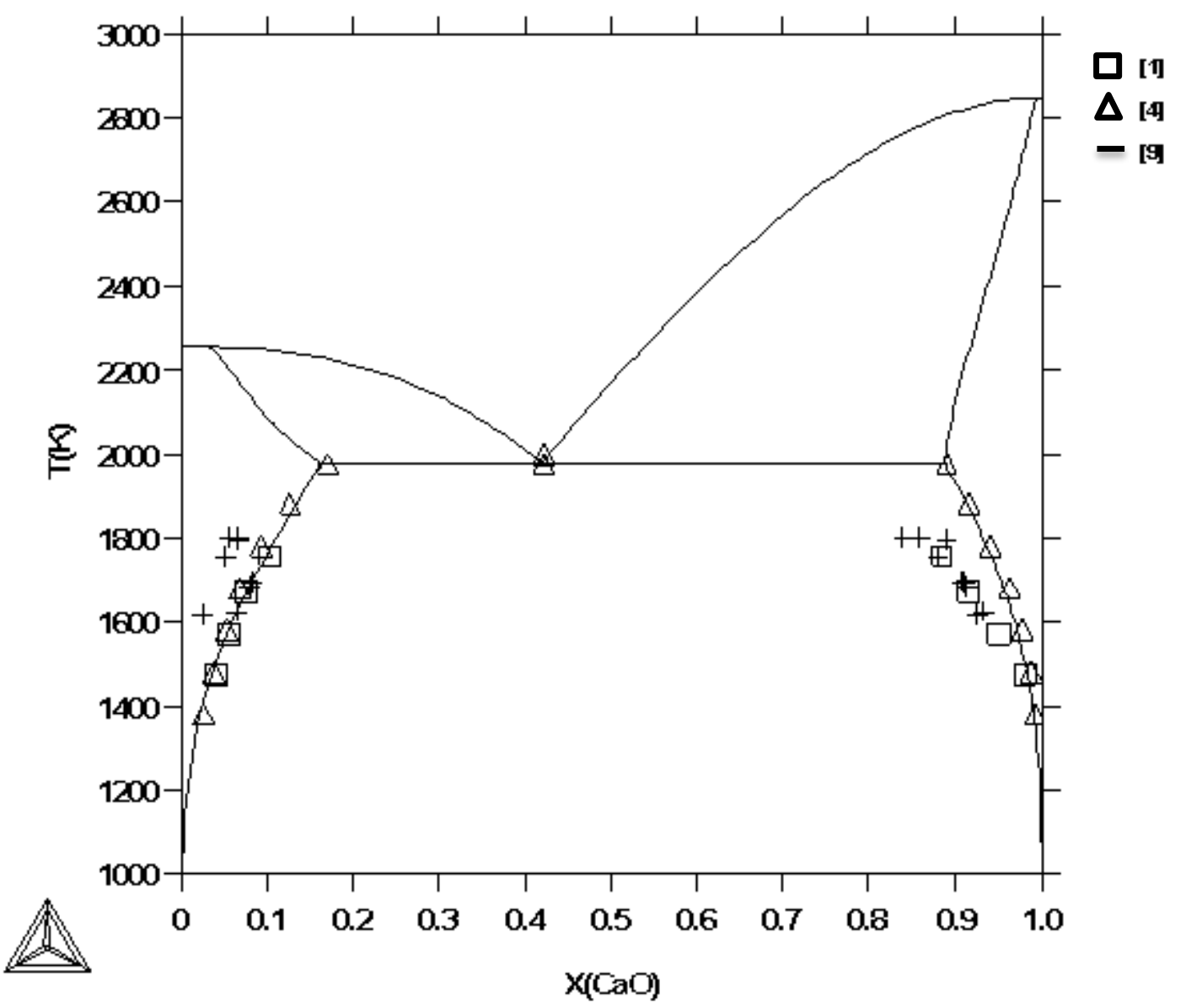

Figura 1. Diagrama de equilíbrio de fases $\mathrm{NiO}-\mathrm{CaO}$ calculado.

A variação da atividade do $\mathrm{CaO}$ com a composição está apresentada na Figura 2. Nela observa-se o melhor ajuste da curva calculada com os dados de Raghavan [3]. Os dados de Rog et al. [2], mesmo tendo sido utilizados na presente otimização, não 
se ajustaram à curva de atividade calculada e, conforme ressaltado por Prostakova et al. [1], impõem, aparentemente, um limite de solubilidade muito maior do que aquele apontado por Smith et al. [4].

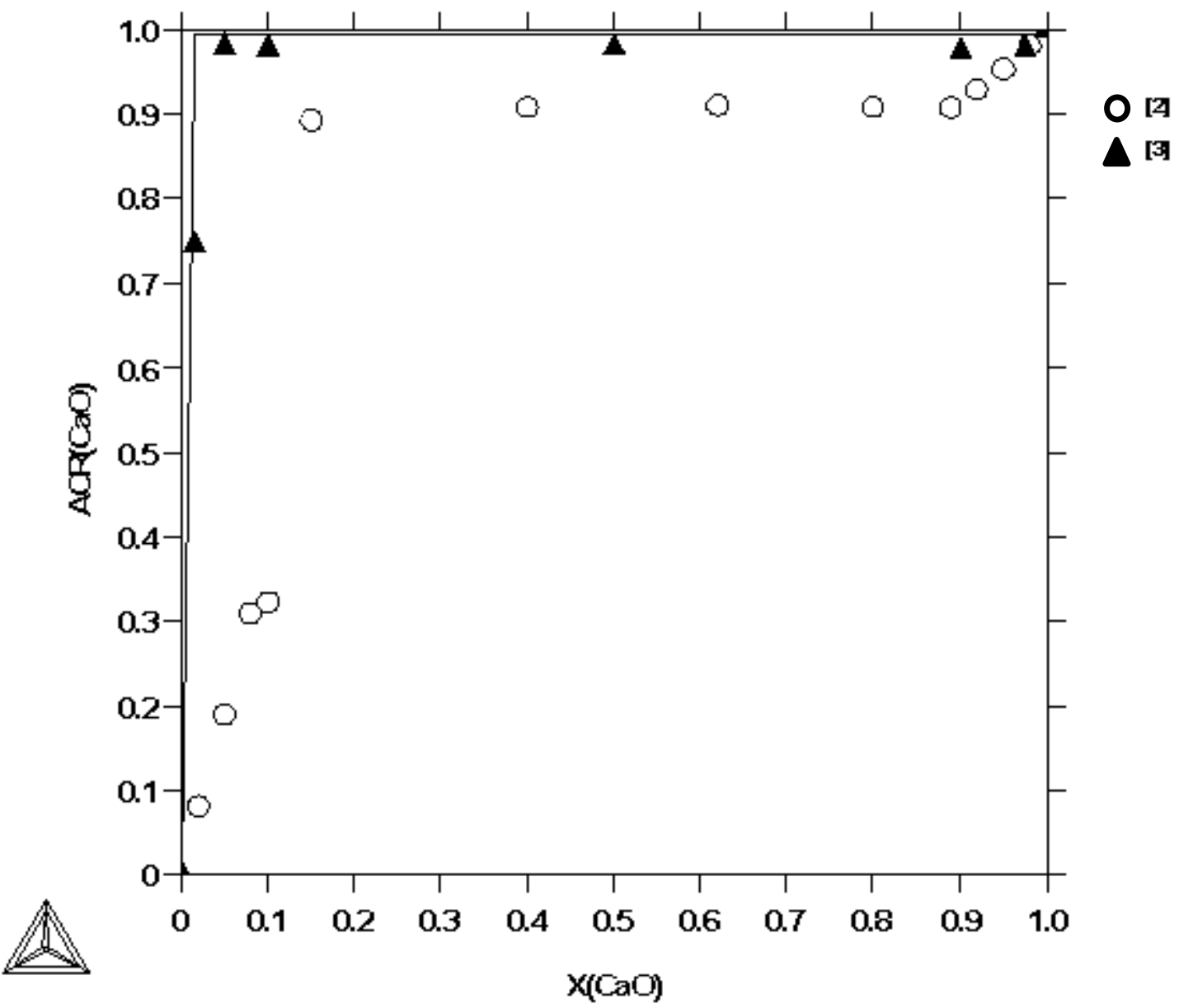

Figura 2. Variação da atividade do $\mathrm{CaO}$ com a composição (ref: $\mathrm{CaO}$ sólido) a 1313K calculada (linha) comparada com dados de literatura (pontos)

\section{CONCLUSÕES}

Foi possível realizar a otimização termodinâmica do líquido do sistema NiO-CaO utilizando o modelo quase-químico de células de Kapoor-Frohberg-Gaye e o sólidos pelo modelo substitucional-regular. Os dados calculados ajustaram-se adequadamente aos dados experimentais.

\section{REFERÊNCIAS}

1. Prostakova, V.; Chenb, J.; Jak, E.; Decterov, S. A. "Experimental study and thermodynamic optimization of the $\mathrm{CaO}-\mathrm{NiO}, \mathrm{MgO}-\mathrm{NiO}$ and $\mathrm{NiO}-\mathrm{SiO}_{2}$ systems".

CALPHAD. 2012; 37:1-10.

2. G. Rog, A. Kozlowska-Rog, M.M. Bucko, J. Chem. Thermodyn. 30 (1) (1998), p.43-47

3. S. Raghavan, J. Mater. Sci. Lett. 7 (4) (1988), p.402-404.

4. D.E. Smith, T.Y. Tien, L.H. Van Vlack, J. Am. Ceram. Soc. 52 (8) (1969), p.459-460.

5. A.D. Pelton, S.A. Decterov, G. Eriksson, C. Robelin, Y. Dessureault, Metall. Mater. Trans. B 31B (4) (2000) 651-659.

6. A.D. Pelton, P. Chartrand, Metall. Mater. Trans. A 32A (6) (2001) 1355-1360 
7. Woo, D.-H.; Lee, H.-G. Jung, I.-H.. "Thermodynamic modeling of the $\mathrm{NiO}-\mathrm{SiO}_{2}, \mathrm{MgO}-$ $\mathrm{NiO}, \mathrm{CaO}-\mathrm{NiO}-\mathrm{SiO}_{2}, \mathrm{MgO}-\mathrm{NiO}-\mathrm{SiO}_{2}, \mathrm{CaO}-\mathrm{MgO}-\mathrm{NiO}$ and $\mathrm{CaO}-\mathrm{MgO}-\mathrm{NiO}-\mathrm{SiO} 2$ systems". Journal of the European Ceramic Society. 2011; 31:43-59.

8. Lukas, H.L.; Fries, S.G.; Sundman, B.. Computational Thermodynamics: The Calphad Method. Cambridge University Press, 2007

9. M. Appel, J. A. Pask. Journal of The American Ceramic Society Vol. 54, No. 3, p.152158

10. Kapoor, M. L.; Frohberg, M. G. Theoritical Treatment of Activities in Silicate Melts. Symposium on Chemical Metallurgy on Iron and Steels. 1971, pp. 17-22.

11. Gaye, H.; Welfringer, J. Second International Symposium on Metallurgical Slags and Fluxes. 1984. Lake Tahoe, Nevada: TMS-AIME., pp. 357-371.

12. DINSDALE, A.T.. SGTE data for pure elements, Calphad, v.15, p.317-425, 1991 